\title{
Una propuesta metodológica para la concepción técnica de micro-redes*
}

\author{
Juan David Mina Casaran* \\ Diego Fernando Echeverry ${ }^{* *}$ \\ Carlos Arturo Lozano Moncada $a^{* * * *}$
}

\author{
Recibido: 03/02/2017 • Aceptado: 27/08/2018 \\ https://doi.org/10.22395/rium.v18n34a12
}

\begin{abstract}
Resumen
En este trabajo se presenta una metodología para la concepción técnica de micro-redes. Se consideran aspectos como el levantamiento de la topología del sistema eléctrico de media tensión, la preselección de tecnologías de fuentes renovables, la priorización de la demanda eléctrica, la localización, el dimensionamiento y las estrategias de operación de los generadores distribuidos, el modelo de componentes y la evaluación de la micro-red. La metodología propone el uso de una técnica de análisis multicriterio para la priorización de la demanda y el índice de estabilidad de voltaje (SI) para la ubicación de generadores distribuidos en la micro-red. En este trabajo se utilizó la herramienta computacional de análisis de sistemas de potencia Neplan ${ }^{\circledR}$ para el modelado de componentes y evaluación del desempeño de la micro-red por medio del índice de regulación de voltaje IREG y la gestión óptima de potencia de las unidades de generación de la micro-red.
\end{abstract}

Palabras clave: calidad de energía; despacho de potencia; generación distribuida; índice IREG; índice SI; micro-red; perfil de tensión.

* Artículo de investigación terminada derivada del proyecto Modelado y simulación de una microgrid con base en el potencial de fuentes renovables no convencionales de energía en Colombia. Tiempo de ejecución: 20142016. Entidades financiadoras: Departamento Administrativo de Ciencia, Tecnología e Innovación Colciencias y Universidad del Valle, Cali, Colombia.

** MSc. en Ingeniería Eléctrica, estudiante de Doctorado en Ingeniería del Grupo de Investigación en Alta Tensión -Gralta, Universidad del Valle, Colombia. Correo electrónico: juan.mina@correounivalle.edu.co. Orcid: http:// orcid.org/0000-0002-9670-6897

*** Doctor en Ingeniería, profesor asociado de la Escuela de Ingeniería Eléctrica y Electrónica, investigador del Grupo de Investigación en Alta Tensión -Gralta, Universidad del Valle, Colombia. Correo electrónico: diego. echeverry@correounivalle.edu.co. Orcid: http://orcid.org/0000-0002-6067-2955

**** Doctor en Ingeniería, profesor asociado de la Escuela de Ingeniería Eléctrica y Electrónica. Decano de la Facultad de Ingeniería, Universidad del Valle, Colombia. Correo electrónico: carlos.a.lozano@correounivalle.edu.co. Orcid: http://orcid.org/0000-0001-5841-1978 


\title{
A methodological proposal for the technical conception of microgrids
}

\begin{abstract}
This paper introduces a methodology for the technical conception of microgrids. It takes into account aspects such as the topological survey of the medium power electrical system, the preselection of renewable sources technologies, the prioritisation of the electrical demand, the localization, dimensioning and operative strategies of the distributed generators, the components model and the microgrid evaluation. This methodology proposes the use of a multi-criteria analysis technique for the prioritisation of the demand and the stability index of (SI) voltage for the location of distributed generators in the microgrid. In this work the computational tool for the power system analysis Neplan ${ }^{\circledR}$ was used for the components modeling and evaluation of the microgrid performance through the IREG voltage regulation index and the optimal power management of the microgrid's generation units.
\end{abstract}

Keywords: power quality; power delivered; distributed generation; IREG index; SI index; microgrid; voltage profile.

\section{Uma proposta metodológica para a concepção técnica de microrredes}

\begin{abstract}
Resumo
Neste trabalho, é apresentada uma metodologia para a concepção técnica de microrredes. São considerados aspectos como o levantamento da topologia do sistema elétrico de média tensão, a pré-seleção de tecnologias de fontes renováveis, a priorização da demanda elétrica, a localização, o dimensionamento e as estratégias de operação dos geradores distribuídos, o modelo de componentes e a avaliação da microrrede. A metodologia propõe o uso de uma técnica de análise multicritério para priorizar a demanda e o índice de estabilidade de voltagem (SI) para localizar geradores distribuídos na microrrede. Neste trabalho, foi utilizada a ferramenta computacional de análise de sistemas de potência Neplan ${ }^{\circledR}$ para o modelado de componentes e avaliação do desempenho da microrrede por meio do índice de regulação de voltagem IREG e da gestão otimizada de potência das unidades de geração da microrrede.
\end{abstract}

Palavras-chave: qualidade de energia; despacho de potência; geração distribuída; índice IREG; índice SI; microrrede; perfil de tensão. 


\section{INTRODUCCIÓN}

Los esquemas tradicionales de generación, transmisión y distribución (GTD) de energía en el mundo, se enfrentan a una serie de retos relacionados con sostenibilidad, eficiencia y escalabilidad [1]. Al tratarse de esquemas centralizados, existen problemas de tipo operacional y ambiental que pueden volver la red eléctrica insostenible y poco eficiente, debido por ejemplo al incremento de pérdidas de energía, congestión de potencia eléctrica e impacto en el medio ambiente por el uso de combustibles fósiles para la generación de electricidad [2]. En muchos países se desarrollan estudios y casos pilotos de redes con generación distribuida (GD) y fuentes no convencionales de energía renovable (FNCER), para evaluar sus fortalezas y debilidades con respecto a las redes actuales con generación centralizada [3-9]. Se espera que estas tendencias energéticas permitan la descentralización de las operaciones de GTD, ahorros en las reservas de combustibles fósiles y reducción en materia de: emisiones de gases contaminantes, costos de expansión de redes de transmisión y distribución, y pérdidas técnicas de energía [10]. Para lograrlo, se precisa una visión compartida de generación, distribución y consumo inteligentes que transforme las redes convencionales en redes con flujo bidireccional de potencia, incorporación de fuentes renovables, cercanía de la generación a los centros de consumo, alto poder de autonomía e intercambio del recurso energético, y que además incluyan conceptos de eficiencia, sostenibilidad, accesibilidad, calidad y confiabilidad energética [11].

Como respuesta tecnológica a las necesidades energéticas citadas, surgen las microredes, las cuales son redes con generación distribuida y gestión de carga, que hacen uso de las tecnologías de la información y comunicación para actuar de manera autónoma o conectada al Sistema Interconectado Nacional (SIN) [12]. Representan un beneficio para los sistemas eléctricos toda vez que brindan mejoras de tipo técnico y ambiental: calidad de la energía, confiabilidad del servicio y seguridad del suministro, así como bajas emisiones de gases contaminantes. No obstante, la implementación de este tipo de redes puede llevar a grandes retos en su concepción y diseño, así como a situaciones problemáticas durante su operación, dado que involucra aspectos relacionados con el potencial energético renovable, la topología actual de los circuitos de media tensión, la selección de tecnologías de generación, los límites de potencia, entre otros aspectos que deben ser tratados. En ese sentido, este artículo propone una metodología con los requerimientos necesarios para concebir una micro-red desde un enfoque técnico, considerando el potencial energético del sitio donde será implementada. El artículo está dividido de la siguiente forma: en la sección 1 se presentan aspectos generales sobre enfoques metodológicos para el diseño de micro-redes. La sección 2 describe la metodología propuesta. En la sección 3 se expone un caso de aplicación de la 
metodología. En la sección 4 se exponen los resultados y análisis sobre el desempeño de la micro-red. Finalmente, en la sección 5 se presentan las conclusiones.

\section{ASPECTOS GENERALES}

Algunos autores presentan metodologías que bien pueden ser aplicadas para la concepción y diseño de micro-redes, teniendo en cuenta características físicas, técnicas y geográficas. A continuación, se presenta una breve descripción de algunos de estos trabajos. En [13] se presenta una metodología para la planeación de una micro-red de servicios públicos. La metodología cuenta con tres etapas: i) preplaneación: se selecciona la región para la implementación de la micro-red teniendo en cuenta aspectos como confiabilidad, precios de electricidad y distancias entre los centros de generación y usuarios, ii) planeación: los generadores son ubicados en la red eléctrica teniendo como objeto reducir las pérdidas técnicas de la red, y iii) estudio de factibilidad: se realiza un análisis costo-beneficio considerando la reducción de emisiones, pérdidas de energía e inversiones de capital.

En [14] se presenta una metodología para la implementación de un sistema de suministro energético para la Universidad de Queensland (Australia), que busca garantizar el suministro continuo de electricidad ante contingencias en la red. La metodología presenta cuatro etapas: i) modelado de la red de media tensión de la universidad, ii) localización de generadores en la red, iii) priorización de la demanda, iv) resincronización de la micro-red.

El Instituto de Investigación de Energía Eléctrica de los Estados Unidos (EPRI), en el trabajo realizado en [15], propone una metodología para evaluar el impacto de la integración de recursos energéticos distribuidos (DER) a los sistemas de potencia. La metodología consiste en cuatro etapas: i) revisión de condiciones del mercado eléctrico para la integración de los DER al sistema de distribución, ii) cuantificación del impacto de los DER sobre el sistema de distribución, iii) evaluación de la confiabilidad en el suministro de potencia, iv) análisis costo-beneficio para medir el impacto de la integración de los DER sobre el sistema de distribución. Por su parte, La investigación realizada en [16] propone una metodología que permite complementar la capacidad de generación de un recurso energético fósil con recursos energéticos renovables con el fin de establecer topologías de micro-redes. La metodología abarca desde la consecución de información del recurso energético a emplear, la evaluación de la demanda, la caracterización técnico-económica de las tecnologías disponibles y la integración de esta información dentro de un sistema de información geográfica. 
En [17] se analizan las estrategias y criterios de suministro y consumo para conseguir que una micro-red opere de manera óptima desde un enfoque técnico-económico. Se muestra cómo se aplicó el procedimiento en una zona aislada de la red Necoclí, Colombia. Las etapas de la metodología son: i) caracterización y cuantificación de requerimientos energéticos en la zona, ii) caracterización y cuantificación del potencial energético renovable, iii) preselección de fuentes de energía viables y estimación de costos, iv) selección de fuentes con la combinación energética óptima.

De manera general, se puede observar que el diseño de micro-redes gira alrededor de tres ejes principales: selección de fuentes de energía, dimensionamiento y ubicación de generadores en la red.

\section{PROPUESTA METODOLÓGICA}

La metodología propuesta para la concepción técnica de una micro-red está orientada a regiones que hacen parte del SIN colombiano y que cuentan con potenciales de generación a partir de FNCER. El proceso metodológico propuesto (figura 1) requiere el desarrollo de cuatro etapas: i) levantamiento de la topología de la red eléctrica de media tensión y preselección de las tecnologías de FNCER, ii) definición de la topología de la micro-red, iii) modelado de componentes y iv) evaluación del desempeño de la micro-red. A continuación, se describen los pasos de cada una de las etapas de la metodología propuesta.

\subsection{Identificación de la topología de la red de media tensión}

Según la Norma Técnica Colombiana NTC 1340-13, las redes de media tensión conforman circuitos rurales o urbanos cuya tensión de operación es, generalmente, $11,4 \mathrm{kV}$ o 13,2 kV [18]. Dichas redes deben contar con elementos que garanticen una operación segura y confiable del servicio de suministro de energía. En la tabla 1 se proponen dos pautas para el levantamiento de la topología de la red eléctrica base.

Tabla 1. Pautas para el levantamiento de la topología de la red base

Inventario de Se realiza un inventario de los elementos en la red: transformadores, líneas, capacitores, componentes reguladores de tensión, interruptores, circuitos laterales, nodos y cargas.

Adecuación y Consiste en ajustar los parámetros de los componentes de la red (resistencia, longitud de asignación de líneas, potencia nominal, entre otros), de acuerdo a los datos reales de la red eléctrica bajo parámetros estudio. 
Paso 1. Identificar la topología del sistema eléctrico de media tensión

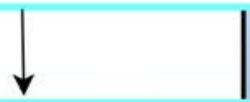

Levantamiento de la topología y preselección de FNCER

Paso 2. Preseleccionar las tecnologías de FNCER

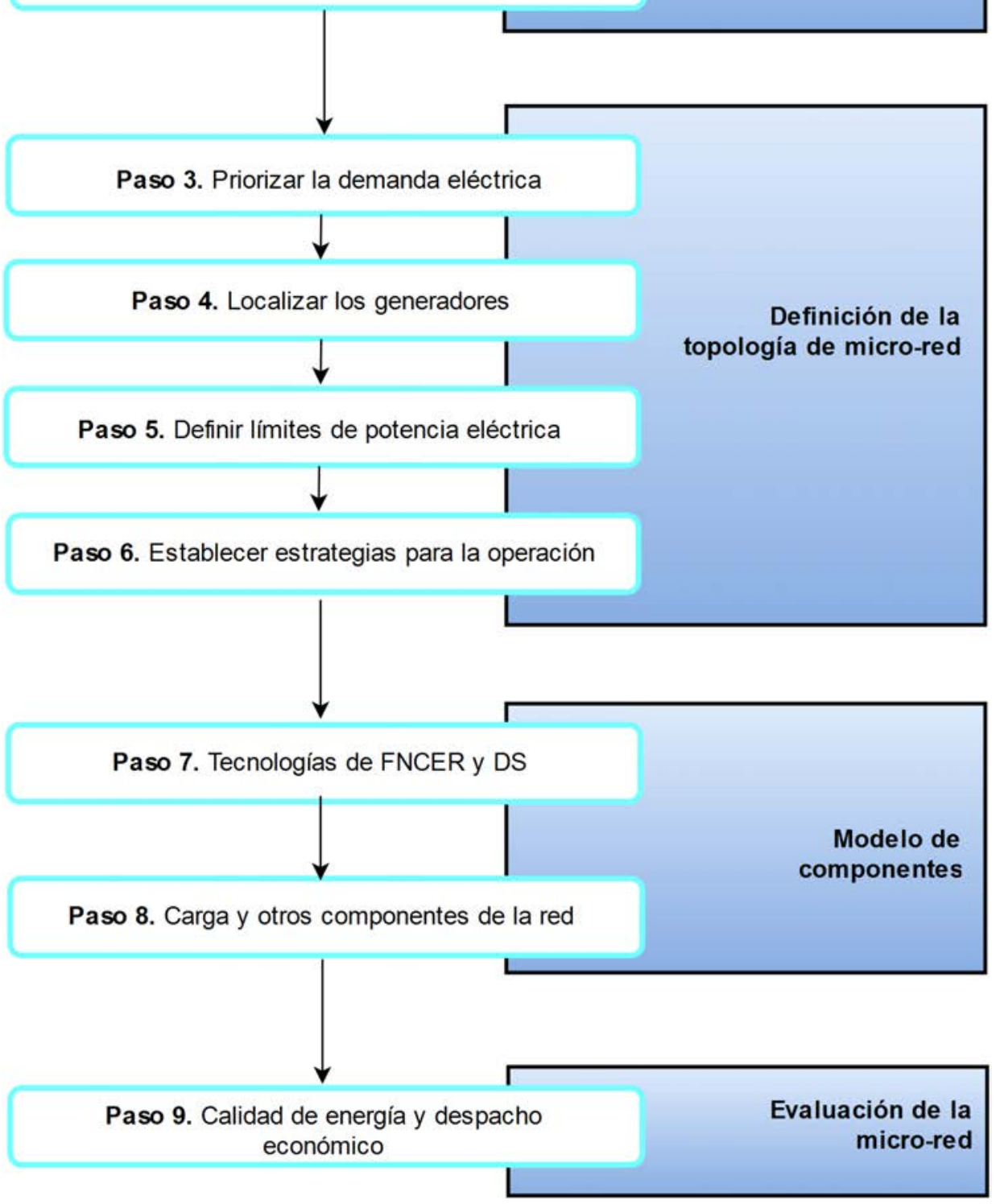

Figura 1. Metodología para la concepción técnica de micro-redes Fuente: elaboración propia. 


\subsection{Preselección de tecnologías de FNCER}

Se realiza en términos de: i) potencial de energías renovables: identificado por medio de los atlas de radiación solar, viento y biomasa [19-22], e información de potencial solar y eólico suministrado por utilities de la región de estudio y ii) características técnicas de las tecnologías existentes de generación. En la tabla 2 se presentan las pautas propuestas para la preselección de las tecnologías de FNCER.

Tabla 2. Pautas para la preselección de las tecnologías de FNCER

Identificar características geográficas y físicas

Identificar el potencial de FNCER

Características de los GD
La instalación de un sistema de generación implica llevar a cabo un estudio de las características climáticas y topográficas: aprovechamientos hidráulicos, emplazamientos para parques eólicos o sombreado en parques solares.

Se produce en términos de las FNCER susceptibles de ser empleadas en la región de interés considerando a su vez los sistemas de almacenamiento de energía.

Escalabilidad, modularidad, eficiencia, uso de combustibles no contaminantes, capacidad de control de voltaje y frecuencia.

Fuente: elaboración propia.

\subsection{Priorización de la demanda}

En la tabla 3 se presentan las pautas para priorizar la demanda, teniendo en cuenta variables cualitativas relacionadas con el tipo de usuario y ubicación en la red, y cuantitativas correspondientes a la potencia de la carga. Las cargas se clasifican como críticas y no críticas o diferibles.

Tabla 3. Pautas para la priorización de la demanda

Potencia de la carga: es un criterio cuantitativo relacionado con la capacidad de la carga $(\mathrm{kW})$.

Definición de criterios

Función o desempeño: representa un criterio de tipo cualitativo relacionado con el rol que desempeña la carga.

Ubicación de la carga en la red eléctrica (circuitos alimentadores): estratificación de los usuarios en la red.

Se realiza un inventario de las cargas teniendo en cuenta los criterios definidos

Inventario de cargas anteriormente.

Asignación de prioridades
Consiste en la asignación de los valores de prioridad a los criterios y a las cargas a través de la escala de importancia del proceso analítico jerárquico (AHP, por sus siglas en inglés) [23].

Cálculo de la prioridad absoluta

Se lleva a cabo la ponderación final, obteniendo matemáticamente la respuesta al encontrar la clasificación de las cargas que son críticas y las que son diferibles. 


\subsection{Localización de generadores}

La idea de encontrar una ubicación fiable de los generadores en la micro-red, surge de la necesidad de determinar un mayor aprovechamiento de la capacidad total de generación disponible en una región determinada y garantizar aspectos técnicos tales como: perfiles de voltaje, reducción de pérdidas de energía y aumento en la capacidad de transferencia de potencia. En este trabajo se propone utilizar el indicador de estabilidad de voltaje (SI, por sus siglas en inglés) definido en [24] y que se expone en (3). A partir de la figura 2 se obtiene las ecuaciones (1) y (2):

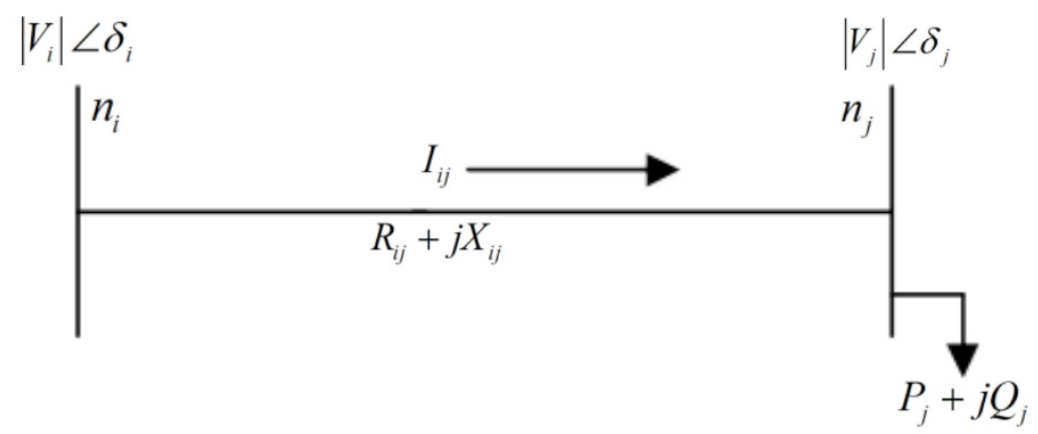

Figura 2. Diagrama esquemático simplificado de una red para la definición del índice SI Fuente: elaboración propia.

$$
\begin{gathered}
I_{i j}=\frac{V_{i}-V_{j}}{R_{i j}+j X_{i j}} \\
P_{j}-j Q_{j}=V_{j}^{*} I_{i j}
\end{gathered}
$$

Donde ni y nj son los nodos de envío y recibo; $i-j$. Iij es la corriente a través de la rama $i-j$; $V i$ y $V j$ son la magnitud del voltaje en $n i$ y $n j$; $P j$ y $Q j$ son la demanda de potencia activa y reactiva en $n j$; Rij y Xij son la resistencia y reactancia de la línea entre ni y $n j$.

Al despejar Iij de la ecuación (2) e igualar con la ecuación (1), se obtiene el índice SI de la ecuación (3):

$$
\operatorname{SI}\left(n_{j}\right)=V_{i}^{4}-4\left(P_{j} R_{i}+Q_{j} X_{i}\right) V_{i}^{2}-4\left(P_{j} X_{i}-Q_{j} R_{i}\right)^{2} \quad \text { [p.u] }
$$

Para localizar los GD en la red, se realiza previamente un flujo de carga en donde se obtiene el perfil de tensiones de los nodos de la red. Posteriormente se evalúa el índice SI y se determinan los nodos con menor índice. Dichos nodos se toman como candidatos para la localización de las unidades de generación. 


\subsection{Límite de potencia de los GD de la micro-red}

Se realiza un modelado matemático del problema no lineal, donde las variables involucradas son los voltajes nodales (magnitud y ángulo) y las potencias (activa y reactiva) que entregan los generadores distribuidos.

Función objetivo: minimizar la diferencia de voltajes que hay en cada nodo con respecto al valor de referencia 1 p.u, tal como se expresa en la ecuación (4):

$$
F O=\sum_{i=1}^{n}\left(V_{i}-V_{\text {ref }}\right)^{2}
$$

Donde Vi es el voltaje en ni; Vref voltaje en el nodo slack; $n$ es el número de nodos.

Restricciones: la función objetivo está sujeta a un conjunto de restricciones técnicas que involucra las ecuaciones de flujo de potencia, límite máximo de generación, límites máximo y mínimo de la magnitud de los voltajes nodales y límites de los ángulos de los voltajes nodales:

- Ecuaciones de flujo de potencia: ecuaciones (5) y (6):

$$
\begin{gathered}
P_{G, i}-P_{D, i}=V_{i} \sum_{j=1}^{n} Y_{i j} V_{j} \cos \left(\delta_{i}-\delta_{j}-\theta_{i j}\right) \\
Q_{G, i}-Q_{D, i}=V_{i} \sum_{j=1}^{n} Y_{i j} V_{j} \operatorname{sen}\left(\delta_{i}-\delta_{j}-\theta_{i j}\right)
\end{gathered}
$$

Donde $P G, i$ y $Q G, i$ son la potencia activa y reactiva generada en $n i ; P D, i$ y $Q D, i$ son la demanda de potencia activa y reactiva en $n i$; $V j$ es la magnitud del voltaje en nj; Yij es la magnitud de la admitancia nodal de la matriz Ybus; $\theta i j$ es el ángulo de la admitancia nodal de la matriz Ybus; $\delta i$ y $\delta j$ son los ángulos del voltaje en los nodos ni y nj.

- Límites de la magnitud de los voltajes nodales: están dados por la ecuación (7), donde Vi,max y Vi,min son los límites máximo y mínimo de voltaje permitido.

$$
\left|V_{i, \min }\right| \leq\left|V_{i}\right| \leq\left|V_{i, \max }\right|
$$

- Límites de los ángulos de los voltajes nodales: están definidos por la ecuación (8), donde $\delta i$,max y $\delta i$,min son los límites máximo y mínimo de los ángulos de los voltajes en los nodos. Usualmente la referencia es el nodo slack $(\delta j=0)$.

$$
\delta_{i, \text { min }} \leq \delta_{i} \leq \delta_{i, \max }
$$


- Límites de potencia de los generadores distribuidos: están dados por las ecuaciones (9) y (10):

$$
\begin{gathered}
P_{G, i \text { min }} \leq P_{G, i} \leq P_{G, i, \text { max }} \\
Q_{G, i, \text { min }} \leq Q_{G, i} \leq Q_{G, i, \text { max }}
\end{gathered}
$$

Donde $P G, i$ y $Q G, i$ son la potencia activa y reactiva generada en $n i ; P G, i, \max$, $P G$,i,min, $Q G, i, \max$ y $Q G, i$, min son los límites máximo y mínimo de potencia activa y reactiva del generador distribuido $i$.

Capacidad del sistema de almacenamiento o respaldo de energía: en este trabajo se propone que la potencia del sistema de almacenamiento o respaldo de energía debe ser mayor o igual a la potencia de la carga crítica del sistema, tal como se describe en la ecuación (11), donde el parámetro PDS,i es la potencia del sistema de almacenamiento o respaldo $i$ y $P D$,crítica es la potencia de la carga crítica.

$$
\sum_{i=1}^{n}\left(P_{D S, i}\right) \geq P_{D, c r i t i c a}
$$

Esta condición aplica para los periodos en donde las fuentes de energía (a excepción del sistema de respaldo o almacenamiento) no se encuentran disponibles para entregar energía a las cargas críticas. A partir de la ecuación (12) se puede calcular el tiempo de autonomía de un banco de baterías según [25].

$$
t=\frac{H}{\left(\frac{I H}{C}\right)^{k}}
$$

Donde $t$ es el tiempo en horas de autonomía de la batería; $H$ es el tiempo de descarga nominal (en horas); I es la corriente de descarga (A); $C$ es la capacidad de la batería (Ah); $k$ es la constante de Peukert (adimensional).

\subsection{Estrategias para la operación}

De acuerdo a las normas IEEE 1547-03 e IEEE 1547.2-08 [25, 26], los generadores a partir de fuentes renovables deben operar con factor de potencia constante, y según los requerimientos de potencia activa y reactiva de los usuarios de la red, los demás generadores pueden operar como generadores de voltaje controlado o de carga. Durante el modo de operación conectado a la red, la energía a partir de las FNCER debe ser utilizada tanto como sea posible para alimentar las cargas. Por su parte, durante el modo 
de operación en isla las fuentes de energía y el sistema de almacenamiento deben ser capaces de suministrar la potencia activa y reactiva a las cargas críticas.

\subsection{Propuesta para el modelado de las tecnologías de FNCER}

Utilizando la herramienta computacional para análisis de sistemas de potencia Neplan ${ }^{\circledR}$, se modelan cada uno de los componentes de la micro-red: generadores, sistemas de almacenamiento, cargas, líneas y transformadores. En la figura 3 se presentan los circuitos ramales de los GD de la micro-red. Cada circuito ramal consta de un convertidor de electrónica de potencia y un transformador que se conecta a la red eléctrica de media tensión.
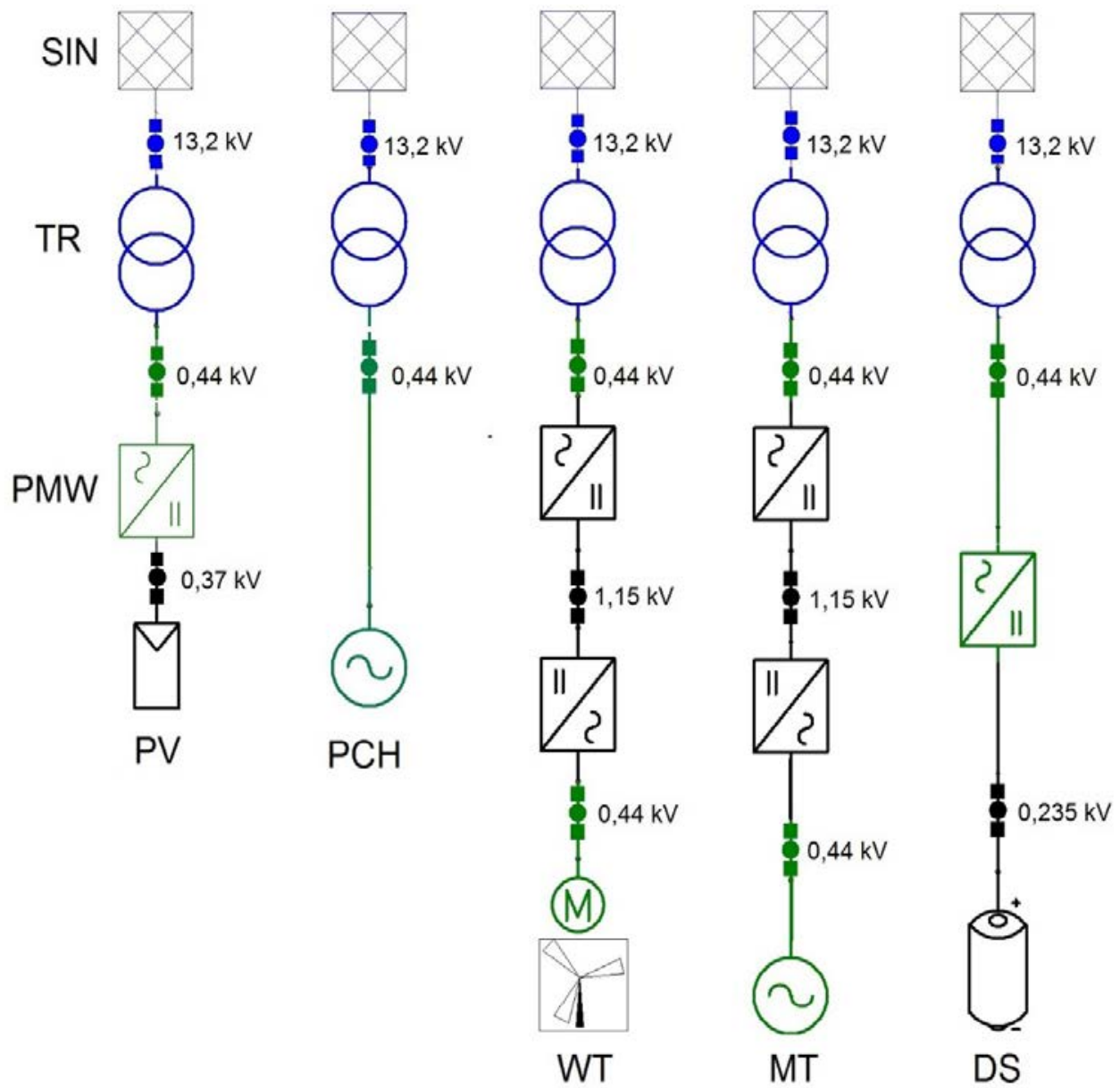

Figura 3. Diagrama esquemático de los circuitos ramales de los GD de la micro-red Fuente: elaboración propia. 
Además del suministro de potencia activa a las cargas de la micro-red, los GD pueden ayudar en labores como: suavización de picos de carga, disminución de pérdidas eléctricas en la micro-red, compensación de reactivos para el control de voltaje, estabilidad de frecuencia y ajuste del factor de carga. Las variables y parámetros de los modelos de los GD se describen en [27-29].

\subsection{Modelo de carga y líneas}

Los circuitos de media tensión están conformados por diferentes tipos de cargas, las cuales presentan diversos comportamientos ante variaciones de potencia, voltaje y corriente. A continuación se presentan tres modelos de carga para análisis de estado estable y dinámico en redes eléctricas: [30-31]:

Modelo de potencia constante: es una típica representación de la carga industrial que contiene gran cantidad de motores. Este modelo es válido para variaciones de voltaje en un rango de 75-120 \% del voltaje nominal.

Modelo de corriente constante: representa la carga comercial, caracterizada por centros de negocios, edificios de oficinas, alumbrado y aires acondicionados.

Modelo de impedancia constante: es una típica representación de la carga residencial caracterizada por elementos como resistencias, calentadores y circuitos de alumbrado.

De manera general, las ecuaciones (13) y (14) representan el comportamiento de los modelos de carga descritos.

$$
\begin{gathered}
P_{\text {oper }}=P_{n}\left(\frac{V_{\text {oper }}}{V_{n}}\right)^{a} \\
Q_{\text {oper }}=Q_{n}\left(\frac{V_{\text {oper }}}{V_{n}}\right)^{b}
\end{gathered}
$$

Potencia constante $(a, b=\mathbf{0})$. Corriente constante $(a, b=\mathbf{1})$. Impedancia constante $(a, b=2)$.

Donde Poper y Qoper son las potencias activa y reactiva de operación; Pn y Qn son las potencias activa y reactiva nominal; Voper es el voltaje de operación y Vn el voltaje nominal.

Modelo de línea: con respecto a las líneas de la micro-red, para su modelado se debe considerar la relación R/X. En la tabla 4 se presentan valores típicos de R y X para líneas de bajo, medio y alto voltaje. 
Tabla 4. Parámetros típicos de una línea por nivel de tensión [33]

\begin{tabular}{lccc}
\hline \multicolumn{1}{c}{ Tipo de línea } & $R(\Omega / \mathrm{km})$ & $X(\Omega / \mathrm{km})$ & $R / X$ \\
\hline Bajo voltaje & 0,642 & 0,083 & 7,7 \\
\hline Medio voltaje & 0,161 & 0,190 & 0,85 \\
\hline Alto voltaje & 0,06 & 0,191 & 0,31 \\
\hline
\end{tabular}

Fuente: elaboración propia.

\subsection{Evaluación de la micro-red}

Calidad de energía: existen diversos índices normalizados para evaluar la calidad de energía en una red eléctrica [32]. En este trabajo se propone evaluar dicho aspecto a través del índice de regulación de tensión (IREG), el cual se presenta en la ecuación (15). El índice IREG evalúa el impacto de la presencia de generación distribuida sobre el perfil de tensiones de la red, en relación con los límites establecidos en la norma NTC 1340-13: tensión máxima nominal de $105 \%$ y mínima de $90 \%$, para tensiones entre $1 \mathrm{kV}$ y $57,5 \mathrm{kV}$.

$$
I R E G=\frac{V_{\text {nodo }}-V_{\text {min }}}{V_{\text {max }}-V_{\text {min }}}
$$

Donde Vnodo es la tensión medida en el punto de conexión común; Vmax y Vmin son los límites de tensión permitidos.

Despacho de potencia: con el objetivo de evaluar la capacidad de gestión sobre los GD de la micro-red, se modelan escenarios de despacho de potencia considerando la disponibilidad de FNCER y el suministro desde el SIN. La función objetivo 1 que se expone en la ecuación (16), minimiza las pérdidas de energía de la micro-red. Dicha función se obtiene de forma teórica a partir de los resultados del problema convencional de flujo de potencia.

$$
F O 1=\sum_{i=1}^{n} \sum_{j=1}^{n}\left[g_{i j}\left(\left|V_{i}\right|^{2}+\left|V_{j}\right|^{2}-2\left|V_{i}\right|\left|V_{j}\right| \cos \left(\delta_{i}-\delta_{j}\right)\right)\right]
$$

Donde $V i$ y $V j$ son la magnitud del voltaje en los nodos $i$ y $j$. Por su parte, gij, $\delta i$ y $\delta j$ son la conductancia de la línea entre los nodos $i-j$, y los ángulos del voltaje en los nodos.

La función objetivo 2 de la ecuación (17), maximiza la potencia total generada por los generadores de la micro-red.

$$
\mathrm{FO} 2=\sum_{i=1}^{n} P_{G, i}
$$

Las restricciones de las funciones descritas corresponden a las ecuaciones (5) a (10). 


\section{APLICACIÓN DE LA METODOLOGÍA A UN CASO DE ESTUDIO}

En la figura 4 se presenta la micro-red concebida al aplicar la metodología propuesta. Como red base se tomó la red IEEE 34 nodos modificada y como región de aplicación con cerca de 500.000 habitantes, zonas agroindustriales y niveles de radiación solar promedio de 4 kWh/día, se seleccionó el municipio de Palmira en el Valle del Cauca, Colombia.

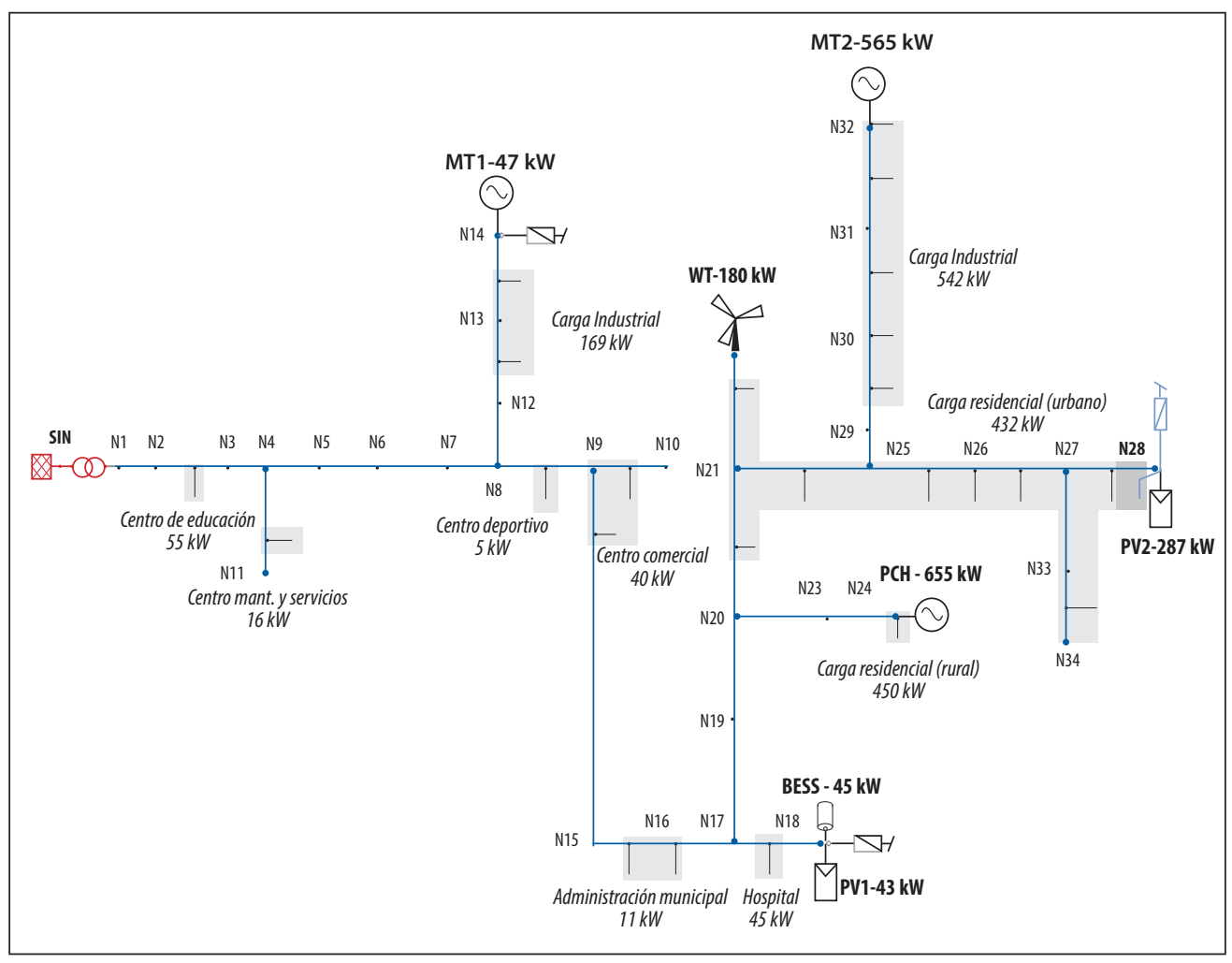

Figura 4. Micro-red concebida a partir de la red IEEE 34N modificada y adaptada al municipio de Palmira, Valle del Cauca

Fuente: elaboración propia

La micro-red se compone de dos sistemas fotovoltaicos PV1-2 de $43 \mathrm{~kW}$ y $287 \mathrm{~kW}$, un aerogenerador WT de $180 \mathrm{~kW}$, dos microturbinas MT1-2 de $47 \mathrm{~kW}$ y $565 \mathrm{~kW}$, y una PCH de $655 \mathrm{~kW}$. La demanda total es $1.765 \mathrm{~kW}$ (25 usuarios). La micro-red también cuenta con tres bancos de reactivos B1-3 de 108 kvar, 350 kvar y 448 kvar. Para el caso de las cargas críticas, se incorporó un banco de baterías (DS) de 50 Ah en hospitales y dos microturbinas de respaldo MTR1-2 de 169 kW y 542 kW, en centros industriales. 


\section{RESULTADOS Y ANÁLISIS}

La evaluación de la calidad de energía se realizó a través del índice IREG. Por su parte, el despacho de potencia de las unidades de generación se llevó a cabo teniendo en cuenta la reducción de pérdidas técnicas de energía y la maximización de la energía suministrada por los GD, desde el entorno de trabajo de la herramienta computacional Neplan $^{\circledR}$. Se analizaron siete casos de estudio (C1 a C7) cuyos resultados fueron comparados con casos base (CB1 y CB2): los casos base corresponden a la micro-red sin GD para las horas del día de mayor demanda: $1.460 \mathrm{~kW}$ al medio día y $1.632 \mathrm{~kW}$ en la noche (horas 13 y 19 respectivamente). En la tabla 5 se presenta una breve descripción de los casos de estudio.

Tabla 5. Casos de estudio para los análisis de calidad de energía y despacho de potencia

\begin{tabular}{|c|c|c|c|c|c|c|c|}
\hline \multirow{3}{*}{ Elemento } & \multicolumn{7}{|c|}{ Modo de operación - Caso de estudio - Estado del elemento: ON/OFF } \\
\hline & \multicolumn{3}{|c|}{$\begin{array}{l}\text { Conectado } \\
\text { (medio dia) }\end{array}$} & \multicolumn{2}{|c|}{$\begin{array}{c}\text { Conectado } \\
\text { (noche) }\end{array}$} & \multirow{2}{*}{$\begin{array}{c}\begin{array}{c}\text { Isla } \\
\text { (medio día) }\end{array} \\
\text { C6 }\end{array}$} & \multirow{2}{*}{$\begin{array}{c}\begin{array}{c}\text { Isla } \\
\text { (noche) }\end{array} \\
\text { C7 }\end{array}$} \\
\hline & $C 1$ & C2 & C3 & C4 & C5 & & \\
\hline PV & ON & OFF & $\mathrm{ON}$ & OFF & OFF & ON & OFF \\
\hline WT & $\mathrm{ON}$ & $\mathrm{OFF}$ & $\mathrm{ON}$ & OFF & OFF & $\mathrm{ON}$ & OFF \\
\hline $\mathrm{PCH}$ & $\mathrm{ON}$ & $\mathrm{OFF}$ & $\mathrm{ON}$ & OFF & OFF & $\mathrm{ON}$ & $\mathrm{ON}$ \\
\hline MT & OFF & $\mathrm{ON}$ & $\mathrm{ON}$ & $\mathrm{ON}$ & ON & ON & ON \\
\hline B1-3 & OFF & OFF & OFF & OFF & OFF & ON & ON \\
\hline MTR & OFF & OFF & OFF & OFF & OFF & OFF & ON \\
\hline DS & \multicolumn{4}{|c|}{ OFF } & $\begin{array}{c}\text { ON } \\
\text { (Generador) }\end{array}$ & $\begin{array}{c}\text { ON } \\
\text { (Generador) }\end{array}$ & $\begin{array}{c}\text { ON } \\
\text { (Generador) }\end{array}$ \\
\hline
\end{tabular}

Fuente: elaboración propia.

\subsection{Calidad de energía}

En la figura 5 se presenta el perfil IREG en los nodos de carga para los casos $\mathrm{Cl}$ a C7 respecto a los casos base CB1 y CB2. Los casos con mejor índice IREG son: C1 (0,58 p.u), C2 (0,42 p.u) y C3 (0,65 p.u), en promedio $13.025 \mathrm{~V}, 12.719 \mathrm{~V}$ y $13.171 \mathrm{~V}$. El caso $\mathrm{C} 3$ presenta el mejor índice IREG, en donde todos los GD suministran energía a los usuarios y además se presentan excedentes de potencia activa que son exportados al SIN. En los casos C4 y C5 no hay suministro de energía por parte de FNCER y, además, el promedio de índice IREG se encuentra por debajo del valor nominal 
(0,67 p.u): 0,36 p.u para C4 y 0,37 p.u para C5. El índice IREG para los casos C6 y C7 equivale a 0,67 p.u (13.205 V) y 0,68 p.u $(12.221 \mathrm{~V})$, respectivamente.

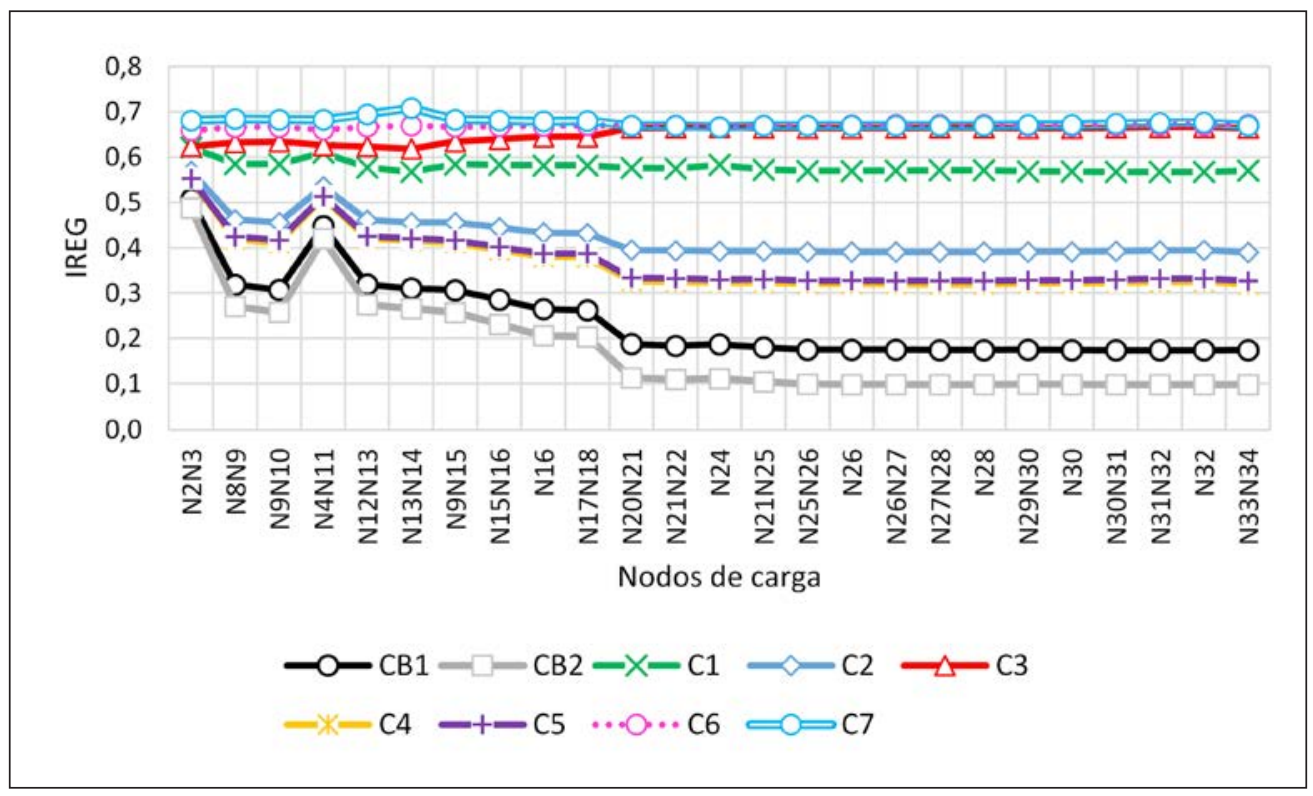

Figura 5. Índice IREG en los nodos de carga para los casos $\mathrm{C} 1$ a C7 Fuente: elaboración propia.

\subsection{Despacho de potencia}

Operación modo conectado: en la figura 6 se presenta la participación energética de las tecnologías de generación cuando la micro-red opera en modo conectado. En el caso C1 el suministro de energía está a cargo de las FNCER y el SIN (30.319 kWh día). En los casos C2 y C4 el suministro es realizado por las microturbinas y el SIN (30.523 kWh día). En el caso C3 todas las fuentes de generación entregan energía a los usuarios (31.640 kWh día); durante este escenario se presentan flujos bidireccionales de potencia entre la micro-red y el SIN: la micro-red entrega al SIN $1.502 \mathrm{kWh}$. En el caso C5, durante las horas pico, los sistemas de almacenamiento DS suministran $180 \mathrm{kWh} /$ día. Por otra parte, las pérdidas totales de energía se reducen respecto al caso base sin GD, pasando de 1.565 kWh día a 225 kWh en C1, 435 kWh en C2 y C4, $46 \mathrm{kWh}$ en $\mathrm{C} 3$ y $428 \mathrm{kWh}$ en $\mathrm{C} 5$. 


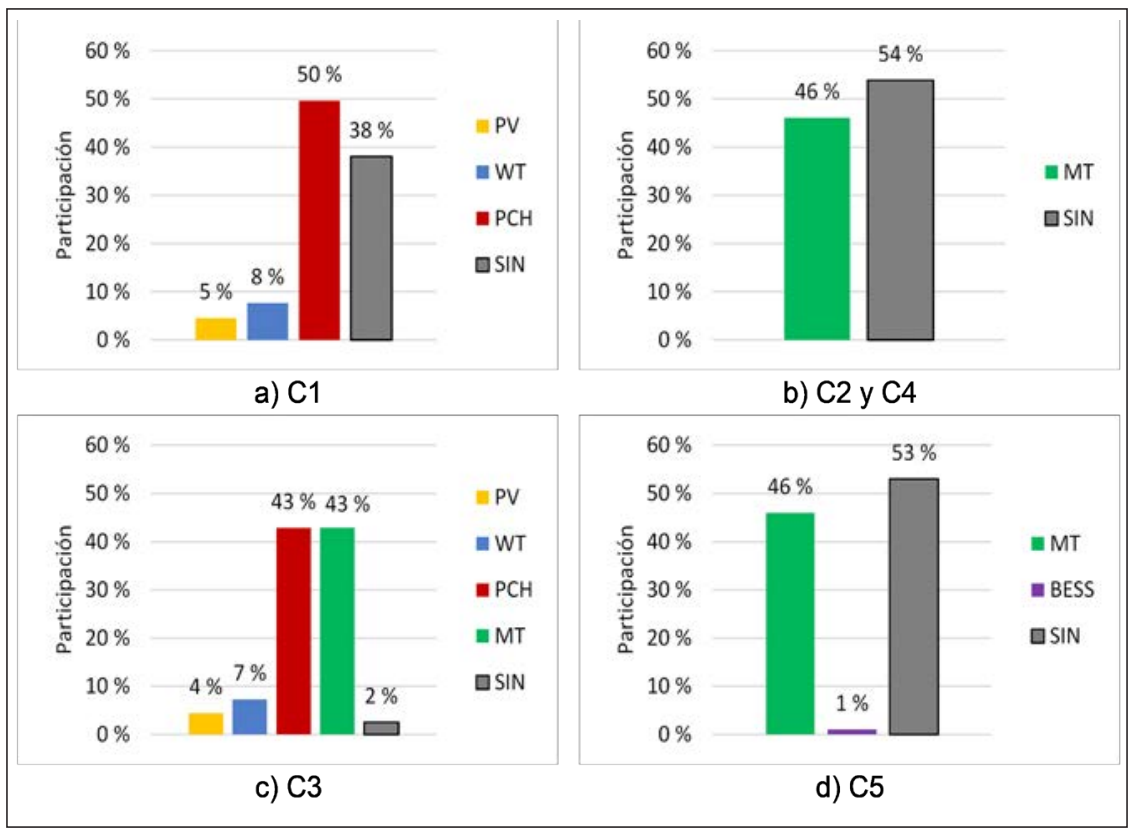

Figura 6. Participación energética de las tecnologías: modo conectado de la micro-red.

Fuente: elaboración propia.

Operación modo isla: en la figura 7 se presenta la participación energética de las tecnologías de generación cuando la micro-red opera en modo isla. En los casos C6 y C7 se utilizó el DS en las horas 13 y 19. El estado de carga del DS se estableció en $100 \%$ de su capacidad. La PCH opera como generador slack. En el caso C6 todos los GD suministran energía a las cargas (30.211 kWh día). En el caso C7 se desconectan los generadores PV y WT y el suministro es asumido por la PCH, las microturbinas y el DS (durante las horas pico). La energía suministrada equivale a $30.203 \mathrm{kWh}$. En los dos casos, las pérdidas totales de energía se reducen respecto al caso base sin GD, pasando de 1.565 kWh día a 115 kWh día.

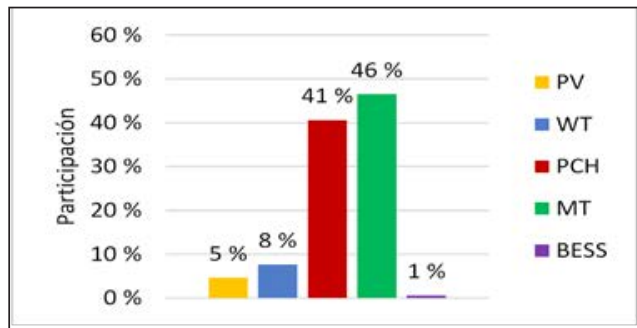

a) $\mathrm{C6}$

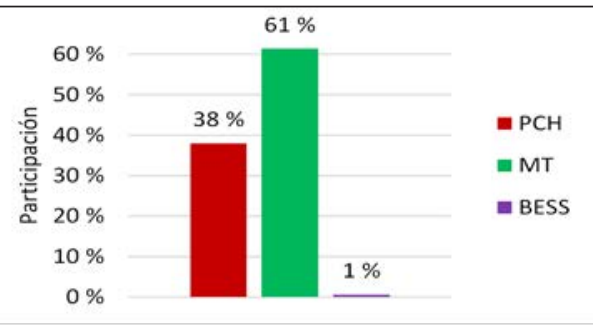

b) $\mathrm{C} 7$

Figura 7. Participación energética de las tecnologías: modo isla de la micro-red Fuente: elaboración propia. 


\section{CONCLUSIONES}

Se presentó una propuesta metodológica para la concepción técnica de micro-redes que considera aspectos de priorización de la demanda, ubicación y dimensionamiento de generadores distribuidos y evaluación del desempeño por medio de simulaciones. La metodología resulta útil en primer lugar para estudios de factibilidad técnica; y en segundo lugar para el diseño de detalle y la optimización técnico-económica de una micro-red. Durante los análisis de calidad de energía, en los casos evaluados mejoró el nivel de tensión de los nodos respecto al de la red base: $\mathrm{C} 3$ fue el caso con mejor promedio de índice IREG (0,65 p.u).

En los análisis de despacho de potencia, las gráficas de participación de energía dan cuenta de la manera cómo el despacho de fuentes alrededor del objetivo minimización de pérdidas ofrece una tendencia a la utilización de las microturbinas y la $\mathrm{PCH}$. Por su parte, el SIN presenta un porcentaje de participación que llega hasta el 50 \% de la energía total cuando no se considera dentro del análisis las FNCER.

En el caso C3 de despacho de potencia se obtuvo la reducción de pérdidas más significativa debido a que el programa de optimización cuenta con el mayor número de generadores gestionables: microturbinas y la $\mathrm{PCH}$.

La metodología desarrollada requirió del modelado y simulación de la micro-red en la herramienta computacional Neplan ${ }^{\circledR}$. Los modelos propuestos en la metodología fueron suficientes para obtener resultados confiables del desempeño de la micro-red.

\section{AGRADECIMIENTOS}

Los autores expresan un sincero agradecimiento al Departamento Administrativo de Ciencia, Tecnología e Innovación -Colciencias- y a la Universidad del Valle por el financiamiento durante la realización del trabajo de grado de maestría titulado Modelado y simulación de una microgrid con base en el potencial de fuentes renovables no convencionales de energía en Colombia.

\section{REFERENCIAS}

[1] S. Tselepis and J. Nikoletatos, "Renewable Energy Integration in Power Grids," IEA-Etsap Irena Technology Brief, 2015. [Online]. Available: www.irena.org.

[2] X. Xia and J. Xia, "Evaluation of Potential for Developing Renewable Sources of Energy to Facilitate Development in Developing Countries," in Asia-Pacific Power and Energy Engineering Conference, 2010, pp. 1-3.

[3] R. Palma-Behnke et al., "A Microgrid Energy Management System Based on the Rolling Horizon Strategy,” IEEE Trans. Smart Grid, vol. 4, no. 2, pp. 996-1006, 2013. 
[4] M. A. Izumida, "Design and Implementation of a Feasible Microgrid Model in Brazil," 2015, pp. 1-9.

[5] J. Hernández, A. M. Blanco, and L. E. Luna, "Design and installation of a smart grid with distributed generation. A pilot case in the Colombian networks," Conf. Rec. IEEE Photovolt. Spec. Conf., pp. 565-569, 2012.

[6] G. Messinis et al., "Multi-microgrid laboratory infrastructure for smart grid applications," MedPower 2014, no. February 2016, pp. 1-6, 2014.

[7] I. Szeidert, I. Filip, O. Prostean, and C. Vasar, "Laboratory setup for microgrid study," INES 2016 - 20th Jubil. IEEE Int. Conf. Intell. Eng. Syst. Proc., pp. 289-292, 2016.

[8] J. L. Espinoza, L. G. Gonzalez, and R. Sempertegui, "Micro grid Laboratory as a Tool for Research on Non-Conventional Energy Sources in Ecuador," 2017 IEEE Int. Autumn Meet. Power, Electron. Comput. Ropec 2017, vol. 2018-Janua, no. Ropec, pp. 1-7, 2018.

[9] C. Patrascu, N. Muntean, O. Cornea, and A. Hedes, "Microgrid Laboratory for Educational and Research Purposes," 2016 IEEE 16th Int. Conf. Environ. Electr. Eng., pp. 1-6, 2016.

[10] T. Foley et al., Renewables 2015 global status report. 2015.

[11] Z. Xue-song, "Research on Smartgrid Technology," in International Conference on Computer Application and System Modeling (Iccasm), 2010, no. Iccasm, pp. 599-603.

[12] J. D. Mina, E. F. Caicedo, and C. A. Lozano, "A proposal of integration of decentralized generation architectures in microgrid environments," Entre Cienc. e Ing., vol. 1, no. 22, pp. 9-17, 2017.

[13] N. Beerea, D. McPhailb, and R. Sharmaa, "A General Methodology for Utility Microgrid Planning," in IEEE PES Asia-Pacific Power and Energy Engineering Conference, 2015, vol. 3, pp. 1-5.

[14] C. Tjah, R. Yan, T. K. Saha, and S. E. Goodwin, "Design Microgrid for a Distribution Network : A Case Study of the University of Queensland," in Power \& Energy Society General Meeting, 2013, pp. 1-5.

[15] Electric Power Research Institute EPRI, "The Integrated Grid a Benefit-Cost Framework," Palo Alto, USA, 2015.

[16] P. A. Manrique Castillo, "Metodología Para el Diseño de Sistemas Híbridos Para Generación de Energía Eléctrica y Análisis de su Viabilidad Mediante el Empleo de un Sistema de Información Geográfica," Universidad del Valle, 2012.

[17] Y. Muñoz and A. Ospino, "Selecting the Optimal Energy Mix and Sizing of a Isolated Microgrid,” Energía y Medio Ambient., vol. 4, no. 7, pp. 59-67, 2013.

[18] Instituto Colombiano de Normas Técnicas y Certificación Icontec, Norma Técnica Colombiana NTC 1340 - Electrotecnia. Tensiones y Frecuencia Nominales en Sistemas de Energía Eléctrica en Redes de Servicio Público. Bogotá, Colombia, 2013. 
[19] Unidad de Planeación Minero Energética UPME, Atlas de Radiación Solar de Colombia. Bogotá, Colombia, 2006.

[20] Unidad de Planeación Minero Energética UPME, Atlas de Viento y Energia Eólica en Colombia. Bogotá, Colombia, 2006.

[21] Unidad de Planeación Minero Energética UPME, Atlas del Potencial Energético de la Biomasa Residual en Colombia. Bogotá, Colombia, 2009.

[22] F. E. Sierra, A. F. Sierra, and C. A. Guerrero, "Pequeñas y microcentrales hidroeléctricas : alternativa real de generación eléctrica,” pp. 8-11, 2011.

[23] T. L. Saaty, "How to Make a Decision: The Analytic Hierarchy Process," Eur. J. Oper. Res., vol. 48, pp. 9-26, 1990.

[24] K. V Kumar and M. P. Selvan, "Planning and Operation of Distributed Generations in Distribution Systems for Improved Voltage Profile," 2009 IEEEPES Power Syst. Conf. Expo., vol. 620015, pp. 1-7, 2009.

[25] A. R. Utomo, "Analysis of Lead Acid Battery Operation Based on Peukert Formula," no. November, pp. 416-419, 2014.

[26] IEEE Standards Coordinating Committee 21, IEEE Application Guide for IEEE Std 1547, Standard for Interconnecting Distributed Resources With Electric Power Systems. New York, USA, 2008.

[27] IEEE Standards Coordinating Committee 21, IEEE Standard 1547 for Interconnecting Distributed Resources with Electric Power Systems. New York, USA, 2003.

[28] M. Z. C. Wanik, A. A. Ibrahim, A. K. M. Hussin, M. R. Rusli, and J. H. Tang, "Simplified Dynamic Model of Photovoltaic Generation System for Grid Integration Studies," in Intelligent and Advanced Systems (Icias), 2014, pp. 1-6.

[29] P. Sorensen, B. Andresen, J. Fortmann, and P. Pourbeik, "Modular Structure of Wind Turbine Models in IEC 61400-27-1,” 2013 IEEE Power Energy Soc. Gen. Meet., pp. 1-5, 2013.

[30] O. Tremblay, L. Dessaint, and A. Dekkiche, "A Generic Battery Model for the Dynamic Simulation of Hybrid Electric Vehicles," in Vehicle Power and Propulsion Conference IEEE, 2007, no. 5, pp. 284-289.

[31] G. Caicedo, C. A. Lozano, A. M. Bahamón, and L. A. Ochoa, "Modelos Para Estimar la Demanda en Sistemas de Distribución,” Energía y Computacion, vol. XI. pp. 35-44, 2002.

[32] D. G. Herrera, G. L. Russi, and E. R. Trujillo, "Evaluación del Impacto de la Generación Distribuida Mediante Índices Normalizados Con Base en la Normatividad Colombiana y Estándares IEEE," Sci. Electron. Libr. Online, vol. 20, no. 2, pp. 299-315, 2015. 